Communications in Physics, Vol. 26, No. 4 (2016), pp. 335-343

DOI:10.15625/0868-3166/26/4/8371

\title{
CALCULATION OF EFFECTIVE EMISSIVITY OF THE CONICAL BASE OF ISOTHERMAL DIFFUSE CYLINDRICAL-INNER-CONE CAVITY USING POLYNOMIAL INTERPOLATION TECHNIQUE
}

\author{
TA VAN TUAN AND NGUYEN QUANG MINH ${ }^{\dagger}$ \\ Center for Systems Engineering and Integration, National Center for Technological Progress \\ ${ }^{\dagger}$ E-mail: lipro.csei@gmail.com \\ Received 03 June 2016 \\ Accepted for publication 10 April 2017
}

\begin{abstract}
We have used the polynomial interpolation technique to calculate effective emissivity of the conical base of isothermal diffuse cylindrical-inner-cone cavities for various cavity parameters such as axial length, aperture radius in the lid and half-angle of the inner cone. Our results are compared with what obtained by other authors using the truncated series approximation technique. The comparative analysis demonstrates considerable accuracy and simplicity of the polynomial interpolation technique. For applications, a series of explicit analytical expressions in the polynomial forms are also presented.
\end{abstract}

Keywords: angle factor, cylindrical-inner-cone cavity, effective emissivity, polynomial interpolation, integral equations.

Classification numbers: 78.20.Ci, 42.79.Gn, 02.60.Ed.

\section{INTRODUCTION}

The artificial blackbody cavities are widely used in metrology as standard sources of thermal radiation. In radiation thermometry, the effective emissivity is often used to evaluate the difference in radiation characteristics between artificial blackbody and ideal one. In practice, the value of effective emissivity of an artificial blackbody should be as identified with ideal one as possible with a relative uncertainty of $0.01 \%$ or less. Since the direct and accurate measurement of effective emissivity is very difficult, therefore the different calculating methods have been used as a primary mode of investigation of blackbody radiation characteristics [1]. Mathematically, the calculation of effective emissivity of blackbody cavity is mostly based on two methods. The first of them is the Integrative Cavity Method (ICM) that describes analytically the radiation exchange between surfaces inside a cavity [2], and the latter is a stochastic method that is also called the Monte-Carlo Method (MCM) using ray tracing algorithm [1-3].

(C)2016 Vietnam Academy of Science and Technology 
The effective emissivity of a cavity depends on its geometry and used materials [1,4]. The calculation of effective emissivity is quite complicated, especially in cases of diffuse cavities. The cylindrical-inner-cone cavity (Fig. 1) is a specific and widely used geometry of artificial blackbody cavities because it provides both high and uniform effective emissivity with a short overall cavity length $[1,5]$. Note that the inner cone causes the obstruction of radiation exchange between cylindrical-wall surfaces creating partial screening effect of internal surface $[1,5,6]$.

In the ICM, the use of integral equations based on the theory of radiative heat transfer with the account of view angle factors is most popularly used in the calculation of effective emissivity of diffuse cavities [1,5-8]. In practice, the exact analytical solutions of such integral equations are limited only for some simple geometrical configurations of cavities. Therefore, the numerical calculation methods have been used in solving those integral equations for diffuse cavities of complex ge-

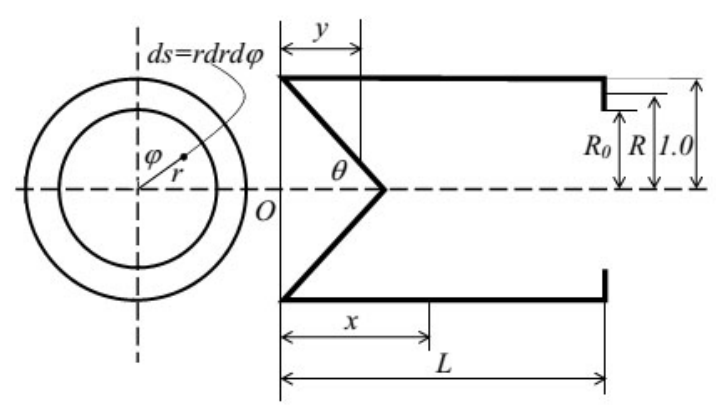

Fig. 1. Geometry of the cylindrical-inner-cone cavity [6]. ometry [1].

Some investigations of cylindrical-inner-cone cavity geometry were previously carried out. Chistyakov et al. [7] provided the analytical expressions in the form of a system of integral equations which serve as the basis for determining the degree of blackness of the cavity model. The emissivity of the cavity in [7] was calculated using universal program written for computer. Chu et al. [5,6] and Berry [8] presented some approximated results obtained by the truncated series approximation technique. However, in the case of nonisothermal cavities, this series method is able to give accurate values only with extreme difficult calculation. The truncated series approximation technique was also applied by Redgrove et al. [9] for a cylindrical-inner-cone cavity in respect to both mixed diffuse and specular reflection conditions. But that work was limited only in treating the points on the conical surface and not those on the cylindrical wall. Note that inaccuracies in the calculations of effective emissivity can occur in cases where singularities exist. The cylindricalinner-cone geometry creates singular points at the apex of a cone, or at a junction of the cavity walls. At some of these points, the view angle factor integrals have an infinite forms which can be treated with the help of L'Hopital's rule [1]. In other cases, the singularities of integral equations may be solved by using zonal approximation technique [10], which transforms the integral equations for effective emissivity into a summation $[1,10]$. The aforementioned techniques are quite difficult to apply because all expressions are complex and /or given in the implicit forms which were calculated with iterative process.

Using the expressions for effective emissivity of the isothermal diffuse cylindrical-innercone cavities given in [6], we have derived the first- and second - order view angle factor terms in the explicit analytical forms and/or in the integral forms. That could make the calculations of effective emissivity simpler than that previously given by single integration using numerical technique [11]. Nevertheless, this technique seems to be still complicated and besides that, the singularities are not evaluated during integration. 
In this paper, we have used the polynomial interpolation technique to calculate effective emissivity of isothermal diffuse cylindrical-inner-cone cavities with the singularities treated by L'Hopital's rule. In particular, effective emissivities of conical base have been calculated for various cavity parameters such as axial length, radius of aperture in the lid and half-angle of the inner cone. Our results are compared with what obtained by other authors using the truncated series approximation technique. The comparative analysis demonstrates considerable accuracy and simplicity of the polynomial interpolation technique. For further applications, a series of explicit analytical expressions in the polynomial forms are also presented.

\section{MATHEMATICAL FORMULATION}

\section{II.1. General Outline}

In this work, we follow the same notations used by Z. Chu in [6] to maintain the meaning of all calculations. Thus, the denotations in Fig. 1 are: $\theta$ - half-angle of inner cone; $L$ - the axial length of the cylindrical portion with unity radius; $x$ - the coordinate of point along the axis of cylinder from the origin $0(0 \leq x \leq L) ; y$ - the coordinate of point along the axis of the inner cone $((0 \leq y \leq 1 / \tan \theta) ; \varphi, r$ - the polar coordinates in the plane of the aperture; $R$ - the coordinate of point along a radius of the lid from the center.

The distribution of the effective emissivity of the conical base can be written in terms of view angle factors, taking into account the diffuse effects [11]. Then, the local emissivity $\varepsilon_{a}\left(y_{0}\right)$ of a certain point with coordinate $y=y_{0}$ on the cone of the interested cavity can be calculated by the following equation:

$$
\varepsilon_{a}\left(y_{0}\right)=1-(1-\varepsilon) d F_{y_{0}, a p}-(1-\varepsilon)^{2} \int_{0}^{L} d^{2} F_{y_{0}, x} d F_{x, a p}
$$

where $\varepsilon$ - intrinsic surface emissivity of the wall of cavity; $d F_{x, a p}$ - the view angle factor between a unit area at position $x$ of the cylinder and the aperture; $d F_{y_{0}, a p}$ - the view angle factor between a unit area at position $y=y_{0}$ and the aperture; $d^{2} F_{y_{0}, x}$ - the view angle factor between a unit area at position $y_{0}$ and an infinitesimal area at position $x$;

Practically, the inner cone should not be too long, so that it will not interfere with diffuse radiation from any unit area of the cylinder to the aperture. The view angle factors $d F_{x, a p}, d F_{y_{0}, a p}$ and $d^{2} F_{y_{0}, x}$ are given by [11]:

$$
\begin{gathered}
d F_{x, a p}=\frac{2}{\pi} \int_{0}^{R} \int_{0}^{\pi} \frac{(L-x)(1-r \cos \varphi)}{\left[(L-x)^{2}+1-2 r \cos \varphi+r^{2}\right]^{2}} r d r d \varphi \\
d F_{y_{0}, a p}=\frac{2}{\pi} \int_{0}^{R} \int_{0}^{\pi} \frac{(L-x)(r \cos \varphi \cos \theta+L \sin \theta-\cos \theta)}{\left[(L-x)^{2}+r^{2}+\left(1-y_{0} \tan \theta\right)^{2}-2 r \cos \varphi\left(1-y_{0} \tan \theta\right)\right]^{2}} r d r d \varphi \\
d^{2} F_{y_{0}, x}=\frac{2}{\pi} \int_{0}^{\phi(x)} \frac{\left[1-\left(1-y_{0} \tan \theta\right) \cos \phi\right](\cos \phi \cos \theta+x \sin \theta-\cos \theta)}{\left[\left(x-y_{0}\right]^{2}+1+\left(1-y_{0} \tan \theta\right)^{2}-2 \cos \phi\left(1-y_{0} \tan \theta\right)\right]^{2}} d \phi d x
\end{gathered}
$$




\section{II.2. Treatment of singularities during view angle factors evaluation}

The view angle factors $d F_{x, a p}, d F_{y_{0}, a p}$ and $d^{2} F_{y_{0}, x}$ can be found in the explicit forms by integrating Eqs. (2)- (4). The evaluation of these integrals is tedious but straightforward by implementing suitable substitutions of variables in the three definite integrals [11]. Finally, we get:

$$
\begin{aligned}
& d F_{x, a p}=\frac{(L-x)}{2}\left[\frac{(L-x)^{2}+R^{2}+1}{\sqrt{(L-x)^{4}+2(L-x)^{2}\left(R^{2}+1\right)+\left(R^{2}-1\right)^{2}}}-1\right], \\
& d F_{y_{0}, a p}=\frac{\cos \theta}{2\left(1-y_{0} \tan \theta\right)\left(L-y_{0}\right)}\left\{\left(L-y_{0}\right)^{2}+\left(1-y_{0} \tan \theta\right)^{2}+\left(1-y_{0} \tan \theta\right)(L \tan \theta-1)\right. \\
& -\frac{\left[\left(L-y_{0}\right)^{2}+\left(1-y_{0} \tan \theta\right)^{2}\right]\left[\left(L-y_{0}\right)^{2}+\left(1-y_{0} \tan \theta\right)^{2}+R^{2}+\left(1-y_{0} \tan \theta\right)(L \tan \theta-1)\right]}{\sqrt{\left[\left(L-y_{0}\right)^{2}+\left(1-y_{0} \tan \theta\right)^{2}+R^{2}\right]^{2}-4\left(1-y_{0} \tan \theta\right)^{2} R^{2}}} \\
& \left.+\frac{\left[2\left(1-y_{0} \tan \theta\right)+(L \tan \theta-1)\right]\left(1-y_{0} \tan \theta\right) R^{2}}{\sqrt{\left[\left(L-y_{0}\right)^{2}+\left(1-y_{0} \tan \theta\right)^{2}+R^{2}\right]^{2}-4\left(1-y_{0} \tan \theta\right)^{2} R^{2}}}\right\} \text {, } \\
& d^{2} F_{y_{0}, x}=\frac{\cos \theta}{\pi} d x\left\{\left[\frac{-8\left(1-y_{0} \tan \theta\right)^{2}[(x \tan \theta-1)-1]}{\left.\left(1-y_{0} \tan \theta\right)\left\langle\left[\left(x-y_{0}\right)^{2}+1+\left(1-y_{0} \tan \theta\right)^{2}\right]^{2}-4\left(1-y_{0} \tan \theta\right)^{2}\right)\right\rangle^{1.5}}\right.\right. \\
& +\frac{4\left(1-y_{0} \tan \theta\right)\left[\left(x-y_{0}\right)^{2}+1+\left(1-y_{0} \tan \theta\right)^{2}\right]\left[(x \tan \theta-1)-2\left(1-y_{0} \tan \theta\right)\right]}{\left(1-y_{0} \tan \theta\right)\left\langle\left[\left(x-y_{0}\right)^{2}+1+\left(1-y_{0} \tan \theta\right)^{2}\right]^{2}-4\left(1-y_{0} \tan \theta\right)^{2}\right\rangle^{1.5}} \\
& \left.+\frac{\left[\left(x-y_{0}\right)^{2}+1+\left(1-y_{0} \tan \theta\right)^{2}\right]^{3}}{\left(1-y_{0} \tan \theta\right)\left\langle\left[\left(x-y_{0}\right)^{2}+1+\left(1-y_{0} \tan \theta\right)^{2}\right]^{2}-4\left(1-y_{0} \tan \theta\right)^{2}\right\rangle^{1.5}}\right] \\
& \times \tan ^{-1}\left[\frac{2\left(1-y_{0} \tan \theta\right)+\left\langle\left(x-y_{0}\right)^{2}+1+\left(1-y_{0} \tan \theta\right)^{2}\right\rangle}{\sqrt{\left[\left(x-y_{0}\right)^{2}+1+\left(1-y_{0} \tan \theta\right)^{2}\right]^{2}-4\left(1-y_{0} \tan \theta\right)^{2}}} \tan \left(\frac{\phi(x)}{2}\right)\right] \\
& +\frac{\left\langle 2-\left[\left(x-y_{0}\right)^{2}+1+\left(1-y_{0} \tan \theta\right)^{2}\right]\right\rangle}{\left\langle\left[\left(x-y_{0}\right)^{2}+1+\left(1-y_{0} \tan \theta\right)^{2}\right]^{2}\right\rangle\left\langle\left[\left(x-y_{0}\right)^{2}+1+\left(1-y_{0} \tan \theta\right)^{2}\right]-2\left(1-y_{0} \tan \theta\right) \cos \phi\right\rangle} \\
& \times \frac{\left\langle 2\left(1-y_{0} \tan \theta\right)(x \tan \theta-1)+\left[\left(x-y_{0}\right)^{2}+1+\left(1-y_{0} \tan \theta\right)^{2}\right]\right\rangle \sin \phi(x)}{\left\langle\left[\left(x-y_{0}\right)^{2}+1+\left(1-y_{0} \tan \theta\right)^{2}\right]^{2}\right\rangle\left\langle\left[\left(x-y_{0}\right)^{2}+1+\left(1-y_{0} \tan \theta\right)^{2}\right]-2\left(1-y_{0} \tan \theta\right) \cos \phi\right\rangle} \\
& \left.-\frac{\phi(x)}{2\left(1-y_{0} \tan \theta\right)}\right\} \text {, } \\
& \text { where } \phi(x)=\left\{\begin{array}{lr}
\pi & x \geq 1 / \tan \theta \\
\pi-\sin ^{-1} \sqrt{1-(x \tan \theta-1)^{2}} & 1 / \tan \theta \leq x \leq 2 / \tan \theta \\
\pi / 2-\sin ^{-1}(1-x \tan \theta) & 0 \leq x \leq 1 / \tan \theta .
\end{array}\right.
\end{aligned}
$$

There are three singular points that require attentions to Eqs. (5), (6) and (7). They have been not treated in our previous work [11]. The first singular point occurs when $x=\mathrm{L}$ and $R=1$ in Eq. (5) when the cavity has no lid. The emissivity of the aperture edge cannot be found directly from Eq. (5). However, by applying L'Hospital's rule, the view angle factor $d F_{x, a p}$ is:

$$
\lim _{\substack{x \rightarrow L^{-} \\ R \rightarrow 1^{-}}} d F_{x, a p}=\frac{1}{2} .
$$


The second singular point occurs when $y=1 / \tan \theta$ in Eqs. (6) and (7). The emissivity of the cone apex cannot also be found directly from the Eq. (6). Similarly, the view angle factors $d F_{y_{0}, a p}$ and $d^{2} F_{y_{0}, x}$ are given by:

$$
\lim _{y_{0} \rightarrow 1 / \tan \theta} d F_{y_{0}, a p}=\frac{\cos \theta}{2}
$$

and

$$
\lim _{y_{0} \rightarrow 1 / \tan \theta} d F_{y_{0}, x}^{2}=\frac{\cos \theta}{\pi} d x \frac{\tan ^{2} \theta\left[\tan ^{2} \theta-(x \tan \theta-1)^{2}\right] \sin \phi(x)}{\left[(x \tan \theta-1)^{2}+\tan ^{2} \theta\right]^{2}} .
$$

The third singular point occurs when $y=L$. This is a singular point mathematically, but not physically because there are no cylindrical-inner-cone cavities with the apex of re-entrance cone sticking out of the aperture, i.e. $y<L$ always. Thus, we neglect this point in our treatment.

After treating the singularities, effective emissivity of the conical base can be now re-written in an explicit and simpler form by introducing Expressions (5)-(10) to Eq. (1). The newly obtained view angle factors can be calculated inherently by numerical integration using trapezoidal rule as used in [11] and the distribution of the conical base effective emissivity could be obtained in the range of $(0 \leq y \leq 1 / \tan \theta)$ with difficult calculation.

\section{II.3. Calculation of the effective emissivity of cavity conical base using polynomial interpo- lation technique}

The second-order terms in $\left((1-\varepsilon)^{2}\right.$ of Eq. (1) have very complicated forms although they are represented in explicit expressions (6) and (7). In practice, a further simplified version of these expressions is required. For this purpose, the polynomial interpolation technique can be better used to represent approximately Expressions (6) and (7).The general formula for an $n$ th-order interpolating polynomial is:

$$
f(y) \simeq P(y)=a_{0} y^{0}+a_{1} y^{1}+a_{2} y^{2}+\ldots+a_{n} y^{n},
$$

where $f(y)$ is an approximated function, $a_{i}(i=0, \ldots, n)$ are the coefficients of the polynomial $P(y)$. For $n+1$ data points, there is one and only one polynomial of order $n$ that passes through all the points. Polynomial interpolation consists of determining the unique $n$ th-order polynomial that fits $n+1$ data points. This polynomial then provides a formula to compute intermediate values.

In our case, the approximated function is chosen:

$$
f\left(y_{0}\right)=\int_{0}^{L} d^{2} F_{y_{0}, x} d F_{x, a p},
$$

its interpolating polynomials have the forms:

$$
P_{f}\left(y_{0}\right)=\sum_{n=0}^{N} a_{n}\left(1-y_{0} \tan \theta\right)^{n} .
$$

As known, the value of effective emissivity of an artificial blackbody should be as identified with ideal one as possible, for example with a relative uncertainty of $0.01 \%$ or less [1]. It means that the interpolation error should be within that uncertainty of $0.01 \%$. In applications, to determine estimating errors, an alternative is to normalize the error to the calculated values itself [12]. In our case, the estimating error for the $n$ th-order interpolating polynomial is equivalent to the difference between the $(n+1)$ th-order and the $n$ th-order prediction. The required value of estimating 
Table 1. Interpolated expressions of Eq. (12) for variuos cavity parameters with the wall intrinsic emissivity is 0.7 .

\begin{tabular}{|c|c|c|c|}
\hline$L$ & $R_{0}$ & $\theta$ & $\left(\int_{0}^{L} d^{2} F_{y_{0}, a p} d F_{x, a p}\right)$ \\
\hline 8 & 0.25 & $30^{\circ}$ & $0.00020418(1-y \tan \theta)^{2}-0.00057577(1-y \tan \theta)+0.00054582$ \\
\hline 8 & 0.25 & $45^{\circ}$ & $0.0000513802(1-y \tan \theta)^{2}-0.00030077(1-y \tan \theta)+0.00047492$ \\
\hline 8 & 0.25 & $60^{\circ}$ & $-0.000012479(1-y \tan \theta)^{2}-0.00016524(1-y \tan \theta)+0.00045043$ \\
\hline 8 & 0.5 & $20^{\circ}$ & $0.00135148(1-y \tan \theta)^{2}-0.00427454(1-y \tan \theta)+0.0030296$ \\
\hline 8 & 0.5 & $45^{\circ}$ & $\mathrm{n} \theta)^{2}-0.00117964(1-\mathrm{y} \tan \theta)+0.0$ \\
\hline 8 & 0.5 & $60^{\circ}$ & $-0.0000502749(1-y \tan \theta)^{2}-0.000648663(1-y \tan \theta)+0.0017787$ \\
\hline 12 & 0.25 & $20^{\circ}$ & $0.0000547286(1-\mathrm{y} \tan \theta)^{2}-0.000143944(1-\mathrm{y} \tan \theta)+0.00013545$ \\
\hline 12 & 0.25 & $30^{\circ}$ & $0.000020462(1-y \tan \theta)^{2}-0.000080629(1-y \tan \theta)+0.00011584$ \\
\hline 12 & 0.25 & $45^{\circ}$ & $3.59008 * 10^{-6}(1-y \tan \theta)^{2}-0.000046335(1-y \tan \theta)+0.00011213$ \\
\hline 12 & 0.5 & $30^{\circ}$ & $0.0000806242(1-\mathrm{y} \tan \theta)^{2}-0.000319012(1-\mathrm{ytan} \theta)+0.00046002$ \\
\hline 12 & 0.5 & $45^{\circ}$ & $0.00001404(1-y \tan \theta)^{2}-0.00018342(1-y \tan \theta)+0.00044535$ \\
\hline 12 & 0.5 & $60^{\circ}$ & $-0.00001682(1-\mathrm{y} \tan \theta)^{2}-0.00010573(1-\mathrm{y} \tan \theta)+0.00044589$ \\
\hline
\end{tabular}

error is used to determinate appropriate order of interpolation [12]. That is, if the interpolating polynomial (12) has an order $N=2$, the normalized interpolation error is within $10^{-5}$. Table 1 presents some explicit expressions of Eq. (12) for various cavity parameters with a wall intrinsic emissivity is 0.7 . We used the second order of interpolation and coefficients $a_{n}$ that were found by elimination method with the known $\left(1-y_{0} \tan \theta\right)^{\prime} s$

The first-order terms $d F_{y_{0}, a p}$ of Eq. (1) written in the explicit forms (6) can be calculated by direct integration as applied in [11] or by polynomial interpolation technique. Our results obtained by integration or interpolation of $d F_{y_{0}, a p}$ are quite usable for calculation of $\varepsilon_{a}\left(y_{0}\right)$ with an estimating error of $10^{-5}$ or less (Table 2). Note that simultaneous use of the interpolation polynomials for the first - and second-order terms at the same time in Eq. (1) gives a possibility to calculate the distribution of effective emissivity $\varepsilon_{a}(y),(0 \leq y \leq 1 / \tan \theta)$ on the conical base.

\section{RESULTS AND DISCUSSIONS}

In all of the examples, the wall emissivity have been chosen to have the same constant value everywhere in the cavity, so the effective emissivity most depends on cavity geometry.

In order to evaluate the accuracy of our obtained results, they have been compared with ones obtained by Z. Chu et al.in [6]. Because their results were mainly presented in average values, we have averaged our values obtained for two view angle factor terms in Eq. (1)and made the comparative analysis shown in Table 2.

Note that in our values of $d F_{y_{0}, a p}$, the reliability of the interpolation-calculated values are also compared with the integration-calculated values. We can see that the two sets of values of $d F_{y_{0}, a p}$ (Table 2) are practically identified and within the uncertainty of $10^{-4}$ or less.

The comparative analysis of the first- and second-order terms of Eq. (1) in Table 2 pointed that our values slightly lower than $\mathrm{Z}$. Chu's ones. The difference may be due to a series of negative terms neglected in Z. Chu calculation [6] using the truncated series approximation technique. However, our and Z. Chu's values are well identified within an uncertainty of $4.10^{-4}$. Noted that 
Table 2. Comparison of the values of the first- and second-order terms of Eq. (1) for various cavity parameters.

\begin{tabular}{|c|c|c|c|c|c|c|c|}
\hline \multirow{2}{*}{$\mathrm{L}$} & \multirow{2}{*}{$R_{0}$} & & $\theta$ & \multicolumn{3}{|c|}{$\left(d F_{y_{0}, a p}\right)_{a v e}$} & \multicolumn{2}{c|}{$\left(\int_{0}^{L} d^{2} F_{y_{0}, a p} d F_{x, a p}\right)_{a v e}$} \\
\cline { 4 - 8 } & & & Integration & Interpolation & Z. Chu [6] & Our resutls & Z. Chu [6] \\
\hline 8 & 0.25 & $30^{\circ}$ & 0.0005498 & 0.000549766 & 0.0006 & 0.00028397 & 0.0003 \\
8 & 0.25 & $45^{\circ}$ & 0.0007318 & 0.000731752 & 0.0008 & 0.00039459 & 0.0004 \\
8 & 0.25 & $60^{\circ}$ & 0.0008689 & 0.000868895 & 0.0009 & 0.000396679 & 0.0004 \\
8 & 0.5 & $20^{\circ}$ & 0.0016611 & 0.00166115 & 0.002 & 0.0012888 & 0.0013 \\
8 & 0.5 & $45^{\circ}$ & 0.002918 & 0.00291794 & 0.0031 & 0.00136147 & 0.0014 \\
8 & 0.5 & $60^{\circ}$ & 0.0034652 & 0.00346522 & 0.0035 & 0.00145737 & 0.0015 \\
12 & 0.25 & $20^{\circ}$ & 0.0001697 & 0.000169667 & 0.0002 & $9.48923 \mathrm{E}-05$ & 0.0001 \\
12 & 0.25 & $30^{\circ}$ & 0.0002342 & 0.000234167 & 0.0003 & $9.41865 \mathrm{E}-05$ & 0.0001 \\
12 & 0.25 & $45^{\circ}$ & 0.0003193 & 0.000319266 & 0.0004 & $9.40906 \mathrm{E}-05$ & 0.0001 \\
12 & 0.5 & $30^{\circ}$ & 0.0009353 & 0.000935335 & 0.001 & 0.00029195 & 0.0003 \\
12 & 0.5 & $45^{\circ}$ & 0.0012753 & 0.00127534 & 0.0014 & 0.000365889 & 0.0004 \\
12 & 0.5 & $60^{\circ}$ & 0.0015318 & 0.00153181 & 0.0016 & 0.000375528 & 0.0004 \\
\hline
\end{tabular}

in [6], Z. Chu et al. confirmed high accuracy of their results obtained with the truncated series approximation technique.

Table 3 shows the average values of the effective emissivity of the conical bases $\left(\varepsilon_{a}\right)_{\text {ave }}$ which were obtained by introducing to Eq. (1) our average interpolated and Z. Chu values presented in Table 2. The differences between these results are within 0.0001 in average.

Table 3. The average effective emissivity of the bases of isothermal diffuse cylindricalinner-cone cavities $\left(\varepsilon_{a}\right)_{\text {ave }}$ for some typical cases and a wall emissivity $\varepsilon=0.7$.

\begin{tabular}{ccccc}
\hline$L$ & $R_{0}$ & $\theta$ & Our techniques & Z. Chu \\
\hline 8 & 0.25 & $30^{\circ}$ & 0.999809515 & 0.999793 \\
8 & 0.25 & $45^{\circ}$ & 0.999744962 & 0.999724 \\
8 & 0.25 & $60^{\circ}$ & 0.999703629 & 0.999694 \\
8 & 0.5 & $20^{\circ}$ & 0.999385678 & 0.999283 \\
8 & 0.5 & $45^{\circ}$ & 0.999002068 & 0.998944 \\
8 & 0.5 & $60^{\circ}$ & 0.998829277 & 0.998815 \\
12 & 0.25 & $20^{\circ}$ & 0.999940559 & 0.999931 \\
12 & 0.25 & $30^{\circ}$ & 0.999921272 & 0.999901 \\
12 & 0.25 & $45^{\circ}$ & 0.999895751 & 0.999871 \\
12 & 0.5 & $30^{\circ}$ & 0.999693123 & 0.999673 \\
12 & 0.5 & $45^{\circ}$ & 0.99958448 & 0.999544 \\
12 & 0.5 & $60^{\circ}$ & 0.999506662 & 0.999484 \\
\hline
\end{tabular}

The first- and second-order terms in Eq. (1) now have explicit and simple forms to calculate. The distributions of $\varepsilon_{a}(y)$ within the range of $(0 \leq y \leq 1 / \tan \theta)$ can be fully calculated by the 


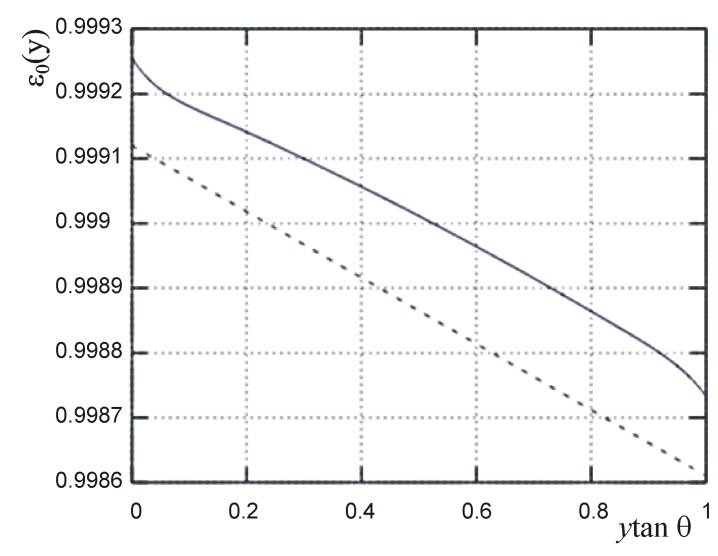

(a) $\theta=30^{\circ}$

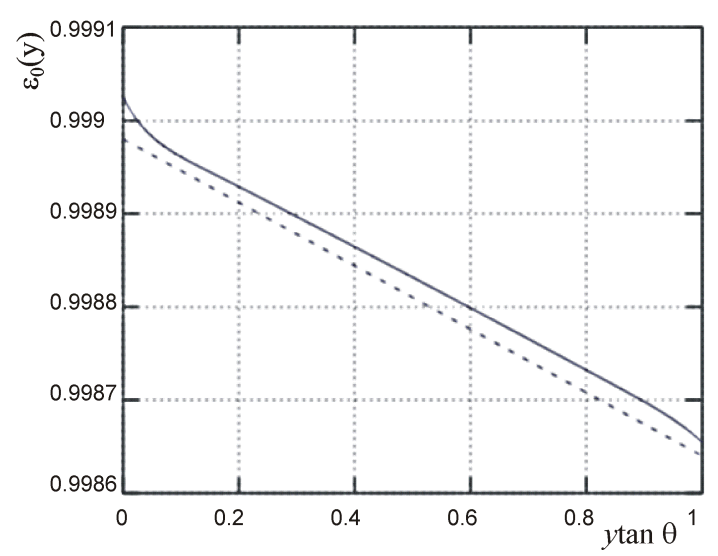

(b) $\theta=45^{\circ}$

Fig. 2. Distributions of the effective emissivity of conical bases of isothermal cylindricalinner-cone cavity with $L=8, R_{0}=0.5, \varepsilon=0.7$. The solid-curves shows the effective emissivity calculated by the present technique and the dashed-curves shows the effective emissivity obtained by Z. Chu's technique [6].

polynomial interpolation technique. For visual comparison, our and Z. Chu's effective emissivity values were plotted in Fig. 2. The curves present the distribution of $\varepsilon_{a}(y)$ in relation with position coordinate, $y \tan \theta$, of cavity base. There are differences in the tendency at the ends of our and Z. Chu curves. These happen due to our treatment of the singularities at $y=0$ and $y=1$. These calculated results well agree with Z. Chu's remarks [6] that the blackest location of cavity is at the junction of the cylinder and the cone, and the less black location is at the apex of the cone.

The comparative analysis demonstrates that such a interpolation is quite suitable and realizable technique of calculating cavity effective emissivity in practice. So, instead of the use of complex expressions in the calculation of effective emissivity, we can use simple expressions obtained by the polynomial interpolation technique. An important difference between our and Z. Chu technique is not to require any iterative process as used by Z. Chu et al. [6]. This facilitates the calculation of effective emissivity and relevant radiation cavity parameters.

\section{CONCLUSION}

In this paper, we present calculations of the effective emissivity of cone base of isothermal diffuse cylindrical-inner-cone cavities using the polynomial interpolation technique. The obtained results demonstrate that the second order of interpolation polynomials is enough for the estimating error of $10^{-5}$. Furthermore, the advantage of this calculating technique is not to require any iterative process. As the rule, such a technique will facilitate the calculations not only for effective emissivity, but also relevant radiation cavity parameters. For real-world applications, a series of explicit analytical expressions in the polynomial forms have been presented.

\section{ACKNOWLEDGMENT}

The authors would like to thank Prof. Dr. Nguyen Dai Hung for inspiring discussion of the present study and for critical remarks. 


\section{REFERENCES}

[1] A. V. Prokhorov, L. M. Hanssen and S. N. Mekhontsev, Experimental Methods in the Physical Sciences 42 (2009) 181.

[2] M. Schalles and G. Blumroeder, Meas. Sci. Technol. 23 (2012) 074023.

[3] A. V. Prokhorov, S. N. Mekhontsev and L. M. Hanssen, Int. J. Thermophys. 28 (2007) 2128.

[4] S. D. Scopatz, J. A. Mazzetta, J. E. Gheiza and M. A. Medina, Comparison of emissivity evaluation methods for infrared sources, Proc. of SPIE, vol. 7300, 2009, pp. 73000Q-1.

[5] Z. Chu, Y. Sun, R. E. Bedford and C. K. Ma, Appl. Opt. 25 (1986) 4343.

[6] Z. Chu, S. Chen and H. Chen, Opt. Soc. Am. 70 (1980) 1270.

[7] V. A. Chistyakov, Z. N. Luzyanina and L. P. Kozlyatnikova, Meas. Tech. 23 (1980) 739.

[8] K. H. Berry, Phys. E. Sci. Instrum. (1981) 629.

[9] J. S. Redgrove and K. H. Berry, High Temp. High Pressure 15 (1983) 1.

[10] R. E. Bedford, M. C.K., C. Z., S. Y. and C. S., Appl. Opt. 24 (1985) 2971.

[11] N. Q. Minh and T. V. Tuan, Evaluation of effective emissivity of a cylinder-inner-cone blackbody simulator cavity, Proc. of the CASEAN-2013, Phnompenh, Cambodia, Publishing House for Science and Technology, 2014, pp. 397-405.

[12] S. C. Chapra and R. P. Canale, Numerical methods for engineers, 6th ed., McGraw Hill, 2010. 